\title{
SYNLETT Spotlight 236 \\ Hydrogen Peroxide: A Versatile Reagent in Organic Synthesis
}

This feature focuses on a reagent chosen by a postgraduate, highlighting the uses and preparation of the reagent in current research

\author{
Compiled by Diego A. Gamba Sanchez
}

Diego Gamba Sanchez was born in Bogotá, Colombia in 1981. He received his B.Sc. in chemistry from the Universidad Nacional de Colombia (Bogotá) in 2004 and his M.Sc. degree in Biomolecules and Organic Synthesis from the Université de Poitiers, France in 2006 under the guidance of Professor Marie-Paule Jouannetaud and Dr. Sébastien Thibaudeau. In 2006 he joined Dr. Joëlle Prunet's research group at Ecole Polytechnique, France where he is currently working towards his $\mathrm{Ph} . \mathrm{D}$. His research is focused on diastereoselective synthesis of 1,3-diols.

Laboratoire de Synthèse Organique, UMR CNRS 7652, Ecole Polytechnique, DCSO, 91128 Palaiseau, France

E-mail: diego.agamba@gmail.com

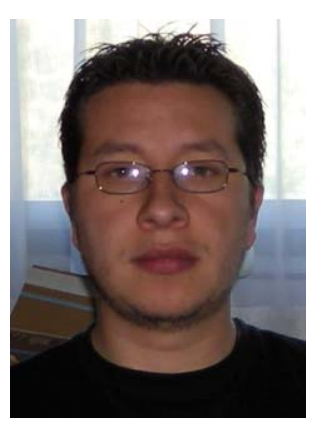

\section{Introduction}

Hydrogen peroxide $\left(\mathrm{H}_{2} \mathrm{O}_{2}\right)$ is a strong oxidizing agent and a weak acid in aqueous solution. It is a very pale blue liquid which appears colorless in dilute solution and is completely miscible with water. Hydrogen peroxide and its highly concentrated aqueous solutions $(>65 \%)$ are soluble in a range of organic solvents, for example carboxylic esters. It decomposes in a violent reaction into water and oxygen if heated above $80{ }^{\circ} \mathrm{C}$. It also decomposes under the influence of light and in the presence of metal ions or oxidizable organic materials. Hydrogen peroxide is com- mercially available in concentrations of $3-90 \%$ as a solution in water.

Hydrogen peroxide and water do not form azeotropic mixtures and can be completely separated by distillation. By fractional crystallization of highly concentrated solutions $100 \%$ pure hydrogen peroxide can be obtained. Pure hydrogen peroxide is usually only of academic interest and is not produced on industrial scale.

Hydrogen peroxide is manufactured by the autoxidation of 2-ethyl-9,10-dihydroxyanthracene to 2-ethylanthraquinone and hydrogen peroxide using oxygen from the air. ${ }^{1}$

\begin{abstract}
s
(A) Hydrogen peroxide is a versatile reagent for the oxidation of sulfides to sulfoxides. Racemic and chiral sulfoxides can be obtained. Zhu and co-workers have reported the asymmetric oxidation of sulfides as well as the kinetic resolution of sulfoxides with hydrogen peroxide in air by a salan-vanadium catalyst; this method provides an efficient procedure for the preparation of chiral sulfoxides in good chemical yields and excellent enantiomeric purities. ${ }^{2}$
\end{abstract}

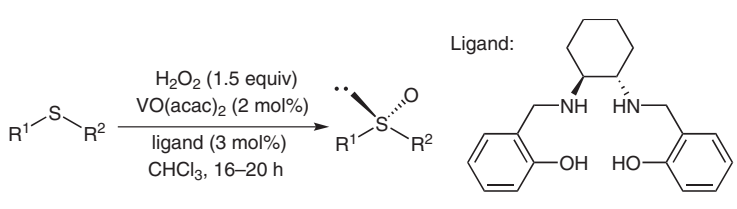

$62-86 \% \quad 51-95 \%$ ee

(B) Hydrogen peroxide has been extensively used for the transformation of alkenes into alcohols. The hydroboration-oxidation sequence constitutes a powerful method for the regio- and stereoselective synthesis of alcohols; usually the $\mathrm{OH}$ group binds to the less-substituted carbon atom (anti-Markovnikoff product). ${ }^{3}$ Besides, Knochel and co-workers used diphosphines as ligands in the rhodium-catalyzed asymmetric hydroboration of styrene derivatives. The Markovnikoff product was obtained in good yield and good enantiomeric excess. ${ }^{3 \mathrm{c}}$

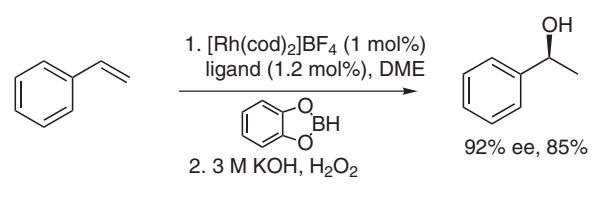

SYNLETT 2008, No. 7, pp 1101-1102

Advanced online publication: 28.03.2008

DOI: 10.1055/s-2008-1066990; Art ID: V24107ST

(c) Georg Thieme Verlag Stuttgart · New York 
(C) Syn-dihydroxylation of alkenes is carried out with hydrogen peroxide in the presence of catalytic osmium tetroxide. ${ }^{4} \mathrm{~A}$ clean and safe method under organic-solvent and metal-free conditions was also reported. ${ }^{5}$ Another way to afford 1,2-diols is a rhodium-catalyzed syn-addition of bis(catecholato)diboron and subsequent oxidation with hydrogen peroxide. ${ }^{6}$

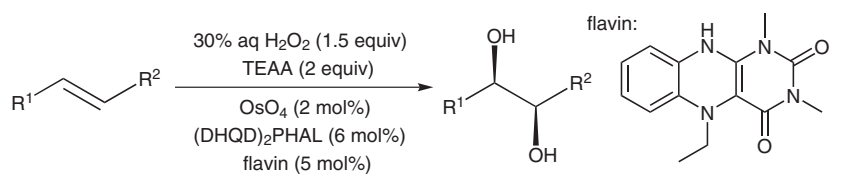

(D) Hydrogen peroxide can be used as oxidizing agent in BaeyerVilliger oxidation to transform ketones into esters or cyclic ketones into lactones. The asymmetric version of this reaction has also been reported using platinum(II) complexes ${ }^{7}$ or a novel planar-chiral bisflavin $^{8}$ as catalysts. An unexpected rearrangement of the initially formed ring-expanded lactone was observed and used in the total synthesis of farnesiferol. ${ }^{9}$

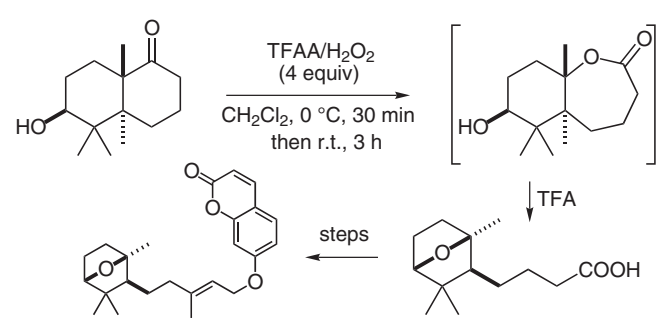

(E) Hydrogen peroxide provides an efficient conversion of alkenes into the respective epoxides. This epoxidation gives the product in good to excellent yields. A range of substrates such as $\alpha, \beta$-unsaturated carbonyl compounds or functionalized olefins has been used. Metallic ${ }^{10}$ or organic ${ }^{11}$ catalysts can be used; it is also possible to car-

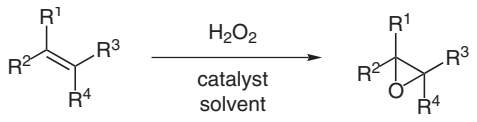
ry out the epoxidation in an enantioselective manner.

(F) The oxidative work-up with hydrogen peroxide after ozonolysis gives carboxylic acids or ketones. Hodgson et al. have successfully used this reaction to complete the stereoselective synthesis of $\mathrm{cis}$ nemorensic acid and 4-hydroxy-cis-nemorensic acid. ${ }^{12}$

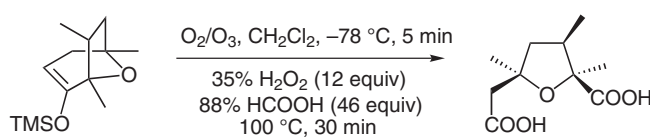

(G) Gopinath and Pate ${ }^{13}$ reported a new catalytic oxidative esterification of aldehydes. This method has advantages over previous methods with respect to the use of environmentally benign catalyst and reagent, cost-effectiveness, high efficiency, mild reaction conditions, shorter reaction times, and facile isolation of the desired products.

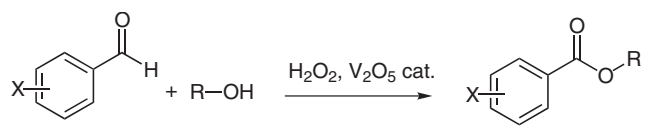

(H) Direct ketohydroxylation can be achieved when olefins are treated with hydrogen peroxide in chloroform containing a catalytic amount of 12-tungstophosphoric acid (WPA, 10\%) combined with cetylpyridiniumchloride (CPC, 30\%) as phase-transfer catalyst at $60{ }^{\circ} \mathrm{C}$. Various $\alpha$-hydroxyketones can be obtained in good yields and

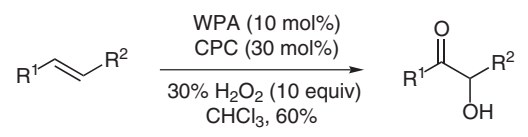
high regioselectivities. ${ }^{14}$

\section{References}

(1) Jones, C. W. Application of Hydrogen Peroxide and Derivatives; Royal Society of Chemistry: Cambridge, 1999.

(2) (a) Karimi, B.; Ghoreishi-Nezhad, M.; Clark, J. H. Org. Lett. 2005, 7, 625. (b) Matteucci, M.; Bhalay, G.; Bradley, M. Org. Lett. 2003, 5 , 5235. (c) Mba, M.; Prins, L. J.; Licini, G. Org. Lett. 2007, 9, 21. (d) Sun, J.; Zhu, C.; Dai, Z.; Xang, M.; Pan, Y.; Hu, H. J. Org. Chem. 2004, 69, 8500.

(3) (a) Clay, J. M.; Vedejs, E. J. Am. Chem. Soc. 2005, 127, 5766. (b) Makabe, H.; Kong, L. K.; Hirota, M. Org. Lett. 2003, 5, 27. (c) Demay, S.; Volant, F.; Knochel, P. Angew. Chem. Int. Ed. 2001, 40, 1235.

(4) Jonsson, S. Y.; Adolfsson, H.; Bäckvall, J.-E. Org. Lett. 2001, 3, 3463.

(5) Usui, Y.; Sato, K.; Tanaka, M. Angew. Chem. Int. Ed. 2003, $42,5623$.

(6) Trudeau, S.; Morgan, J. M.; Shrestha, M.; Morken, J. P. J. Org. Chem. 2005, 70, 9538.
(7) (a) Sgarbossa, P.; Scarso, A.; Michelin, R. A.; Strukul, G. Organometallics 2007, 26, 2714. (b) Paneghetti, C.; Gavagnin, R.; Pinna, F.; Strukul, G. Organometallics 1999, $18,5057$.

(8) Murahashi, S.; Ono, S.; Imada, Y. Angew. Chem. Int. Ed. 2002, 41, 2366.

(9) Demnitz, F. W.; Philippini, C.; Raphael, R. A. J. Org. Chem. 1995, 60, 5114.

(10) (a) Lane, B. S.; Vogt, M.; DeRosa, V. J.; Burgess, K. J. Am. Chem. Soc. 2002, 124, 11946. (b) Tong, K.-H.; Wong, K.-Y.; Chan, T. H. Org. Lett. 2003, 5, 3423.

(11) Marigo, M.; Franzen, J.; Poulsen, T. B.; Zhuang, W.; Jørgensen, K. A. Org. Biomol. Chem. 2005, 3, 3883.

(12) Hodgson, D. M.; Avery, T. D.; Donohue, A. C. Org. Lett. 2002, 4, 1809.

(13) Gopinath, R.; Patel, B. Org. Lett. 2000, 2, 577.

(14) Zhang, Y.; Shen, Z.; Tang, J.; Zhang, Y.; Kong, L.; Zhang, Y. Org. Biomol. Chem. 2006, 4, 1478. 